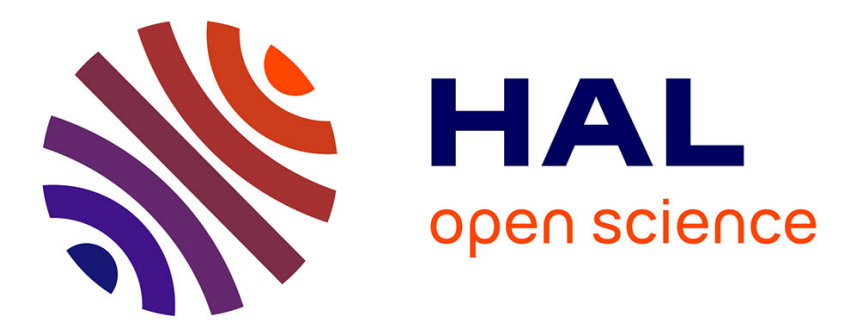

\title{
Influence of myelin proteins on the structure and dynamics of a model membrane with emphasis on the low temperature regime
}

\author{
W Knoll, Judith Peters, P Kursula, Y Gerelli, F Natali
}

\section{To cite this version:}

W Knoll, Judith Peters, P Kursula, Y Gerelli, F Natali. Influence of myelin proteins on the structure and dynamics of a model membrane with emphasis on the low temperature regime. Journal of Chemical Physics, 2014, 141 (20), pp. 205101. 10.1063/1.4901738 . hal-01582610

\section{HAL Id: hal-01582610 https://hal.science/hal-01582610}

Submitted on 6 Sep 2017

HAL is a multi-disciplinary open access archive for the deposit and dissemination of scientific research documents, whether they are published or not. The documents may come from teaching and research institutions in France or abroad, or from public or private research centers.
L'archive ouverte pluridisciplinaire HAL, est destinée au dépôt et à la diffusion de documents scientifiques de niveau recherche, publiés ou non, émanant des établissements d'enseignement et de recherche français ou étrangers, des laboratoires publics ou privés. 


\section{AD| $\begin{aligned} & \text { The Journal of } \\ & \text { Chemical Physics }\end{aligned}$}

Influence of myelin proteins on the structure and dynamics of a model membrane with emphasis on the low temperature regime

W. Knoll, J. Peters, P. Kursula, Y. Gerelli, and F. Natali

Citation: The Journal of Chemical Physics 141, 205101 (2014); doi: 10.1063/1.4901738

View online: http://dx.doi.org/10.1063/1.4901738

View Table of Contents: http://scitation.aip.org/content/aip/journal/jcp/141/20?ver=pdfcov

Published by the AIP Publishing

\section{Articles you may be interested in}

Non-thermal mechanism of weak microwave fields influence on neurons

J. Appl. Phys. 114, 104701 (2013); 10.1063/1.4821027

Relationship between disease-specific structures of amyloid fibrils and their mechanical properties Appl. Phys. Lett. 102, 011914 (2013); 10.1063/1.4774296

C $13-$ C 13 and N 15 - C 13 correlation spectroscopy of membrane-associated and uniformly labeled human immunodeficiency virus and influenza fusion peptides: Amino acid-type assignments and evidence for multiple conformations

J. Chem. Phys. 128, 052319 (2008); 10.1063/1.2829984

Simple model of membrane proteins including solvent

J. Chem. Phys. 124, 184904 (2006); 10.1063/1.2193511

Prediction of membrane protein structures by replica-exchange Monte Carlo simulations: Case of two helices J. Chem. Phys. 120, 10837 (2004); 10.1063/1.1712942

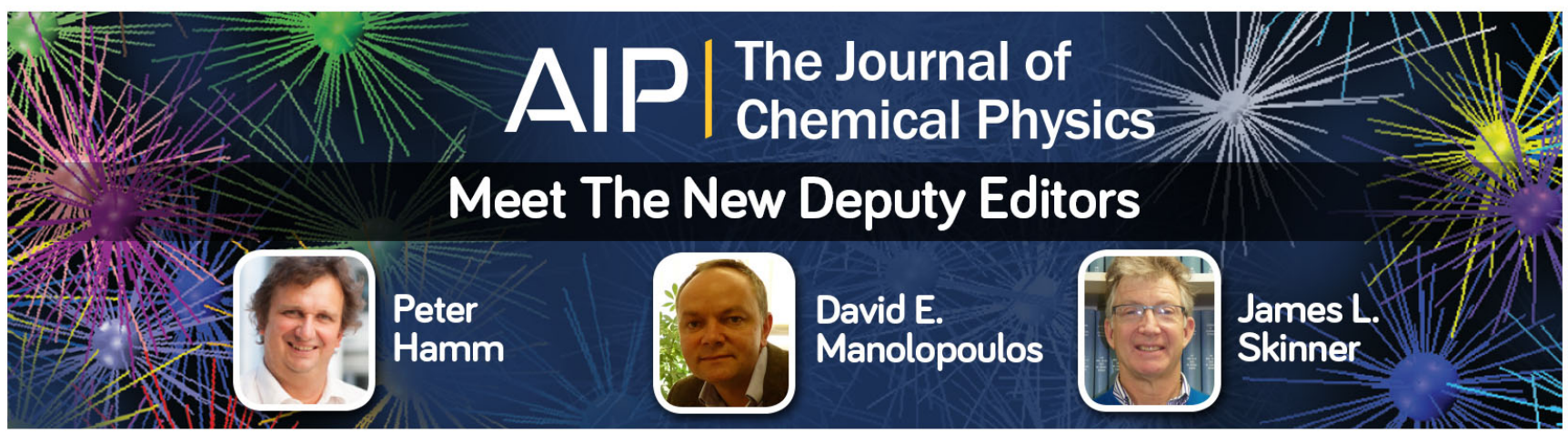




\title{
Influence of myelin proteins on the structure and dynamics of a model membrane with emphasis on the low temperature regime
}

\author{
W. Knoll, ${ }^{1,2}$ J. Peters, ${ }^{1,2,3}$ P. Kursula, ${ }^{4,5}$ Y. Gerelli, ${ }^{2}$ and F. Natali ${ }^{2,6, a)}$ \\ ${ }^{1}$ University Joseph Fourier, UFR PhiTEM, Grenoble, France \\ ${ }^{2}$ Institut Laue-Langevin, Grenoble, France \\ ${ }^{3}$ Institut de Biologie Structurale, Grenoble, France \\ ${ }^{4}$ University of Oulu, Oulu, Finland \\ ${ }^{5}$ CSSB-HZI, DESY, Hamburg, Germany \\ ${ }^{6} \mathrm{CNR}-I O M-O G G$, c/o Institut Laue-Langevin, Grenoble, France
}

(Received 23 October 2014; accepted 24 October 2014; published online 24 November 2014)

\begin{abstract}
Myelin is an insulating, multi-lamellar membrane structure wrapped around selected nerve axons. Increasing the speed of nerve impulses, it is crucial for the proper functioning of the vertebrate nervous system. Human neurodegenerative diseases, such as multiple sclerosis, are linked to damage to the myelin sheath through demyelination. Myelin exhibits a well defined subset of myelin-specific proteins, whose influence on membrane dynamics, i.e., myelin flexibility and stability, has not yet been explored in detail. In a first paper [W. Knoll, J. Peters, P. Kursula, Y. Gerelli, J. Ollivier, B. Demé, M. Telling, E. Kemner, and F. Natali, Soft Matter 10, 519 (2014)] we were able to spotlight, through neutron scattering experiments, the role of peripheral nervous system myelin proteins on membrane stability at room temperature. In particular, the myelin basic protein and peripheral myelin protein 2 were found to synergistically influence the membrane structure while keeping almost unchanged the membrane mobility. Further insight is provided by this work, in which we particularly address the investigation of the membrane flexibility in the low temperature regime. We evidence a different behavior suggesting that the proton dynamics is reduced by the addition of the myelin basic protein accompanied by negligible membrane structural changes. Moreover, we address the importance of correct sample preparation and characterization for the success of the experiment and for the reliability of the obtained results. () 2014 AIP Publishing LLC. [http://dx.doi.org/10.1063/1.4901738]
\end{abstract}

\section{INTRODUCTION}

The myelin sheath is a tightly packed multi-layered membrane wrapped around selected nerve axons in both the central (CNS) and the peripheral (PNS) nervous system. Whereas in the CNS, each oligodendrocyte may myelinate segments of several axons, ${ }^{1}$ in the PNS, a Schwann cell myelinates only one segment of an axon. ${ }^{2,3}$ Myelin is a lipid-rich membrane (75\%-80\% lipids, $20 \%-25 \%$ proteins $^{4}$ ) with a low water content, which allows electrical insulation of the axons. Its unique segmental structure causes jumps of the nerve impulse from node to node by a process called saltatory conduction. Therefore, the nerve impulse conduction is very fast (up to $100 \mathrm{~m} / \mathrm{s}$ in a healthy person). ${ }^{3}$ In demyelinating diseases, such as $m u l$ tiple sclerosis (MS), occurring in the CNS, or the GuillainBarré syndrome in the PNS, damage to the myelin sheath results in ineffective signal conduction by the axons.

Myelin consists of cholesterol, phospholipids, glycolipids, ${ }^{5}$ and a well defined subset of myelin-specific proteins, ${ }^{6}$ which have unique biochemical properties and interact closely with the membrane. The major CNS myelin proteins are the myelin basic protein (MBP) and the proteolipid protein (PLP), which constitute $80 \%$ of the total protein fraction. ${ }^{3}$ The PNS myelin contains, for example,

\footnotetext{
a) Author to whom the correspondence should be addressed. Electronic mail: natali@ill.fr
}

MBP, the P0 glycoprotein, the peripheral myelin protein 2 (P2), and the peripheral myelin protein-22 (PMP-22). Atomic force microscopy (AFM) studies have recently shown that MBP and P2, the only two non-transmembrane proteins in compact myelin, act synergistically to cause stacking of lipid bilayers. ${ }^{7}$

MBP is one of the main agents responsible for the formation and compaction of CNS myelin ${ }^{8,9}$ and plays a structural role in maintaining myelin stability. ${ }^{10}$ The most abundant isoform of MBP in the adult human brain is a $18.5-\mathrm{kDa}$ protein, representing $30 \%$ of the total protein fraction in the $\mathrm{CNS}^{11}$ and $5 \%-18 \%$ in the PNS myelin membrane. ${ }^{12}$ Due to its high positive charge and low hydrophobicity, which lead to intramolecular electrostatic repulsion, MBP exists in an extended, intrinsically disordered, conformation in solution. ${ }^{13-15}$ It binds to negatively charged lipids and maintains the adhesiveness of the cytosolic surfaces of the multilamellar myelin sheath. ${ }^{16,17}$ Its interactions with various cytosolic proteins imply a function as a binding agent and a scaffolding protein. ${ }^{17} \mathrm{MBP}$ is one of the auto-antigens in $\mathrm{MS}^{18,19}$ and in autoimmune encephalomyelitis (EAE), which serves as an experimental animal model for MS. ${ }^{20}$

The peripheral myelin protein $\mathrm{P} 2$ is only present in the PNS, where it constitutes $1 \%-15 \%$ of the protein fraction ${ }^{5,21}$ and locates on the cytoplasmic side of compact myelin. ${ }^{22}$ This $14.5-\mathrm{kDa}$ protein belongs to the family of fatty acid binding proteins ${ }^{23}$ and is thought to play a similar structural 
role in PNS myelin as MBP does in CNS myelin. Due to its lipid-binding activity, $\mathrm{P} 2$ is supposed to interact with specific myelin lipids, maintaining the myelin structure. The crystal structures of human, ${ }^{24}$ bovine and equine P2 has been established by X-ray crystallography, ${ }^{25,26}$ showing a compact 10 -stranded antiparallel $\beta$-barrel. P2 is likely to be one of the auto-antigens in Guillain-Barré syndrome, ${ }^{27,28}$ an autoimmune disease of the PNS.

Circular dichroism (CD) and X-ray scattering experiments have been used to show that the structure of MBP and P2 is affected, when the proteins are bound to lipids. Lipidfree MBP is largely disordered, while lipid-bound MBP has an ordered structure with $\beta$-sheet and $\alpha$-helix regions. ${ }^{29,30} \mathrm{In}$ vestigation of the MBP structure by transmission electron microscopy and computational analysis on single MBP particles purified from bovine brain and adsorbed to a lipid monolayer, led to a three-dimensional model of a MBP-lipid complex with MBP being a "C"'-shaped, folded, although not globular, protein. $^{31,32}$ Also the structure of $\mathrm{P} 2$ is influenced by lipids, which causes a transformation of an $\alpha$-helix motif to a $\beta$-sheet structure. $^{33}$

To better understand the still unknown etiology of inflammatory demyelinating diseases, the determination of detailed structure and dynamics of myelin and the influence of myelin proteins therein are essential. Wide-angle X-ray scattering studies have demonstrated that the lipid phase transition temperature of MS patient myelin was approximately $20^{\circ} \mathrm{C}$ lower than that of healthy myelin, which indicates differences in the structure of the myelin bilayer. ${ }^{34}$ With electron microscopy, it was shown that MBP induces a structural transition of micelles to multi-lamellar vesicles, suggesting that MBP is able to organize myelin lipids. ${ }^{8,35}$ Further AFM studies on myelin model membranes indicated that non-covalent, mainly electrostatic and hydrophobic, interactions are responsible for the multi-lamellar structure and stability of myelin and that MBP acts as a lipid-coupler between two apposed bilayers. Optimal myelin adhesion and stability depend, thus, on the amount and ratio of the lipid and protein charges. ${ }^{36}$

Besides structural properties, dynamical behaviour is also crucial to the correct functioning of biomolecules. Typical membrane dynamics occur in a time-window ranging from $10^{-12} \mathrm{~s}$, corresponding to chain defect motions of the lipid chains, to $1 \mathrm{~s}$, according to collective undulations of the whole membrane. ${ }^{37,38}$ Incoherent elastic (ENS) and quasielastic (QENS) neutron scattering cover the time regime between $10^{-10} \mathrm{~s}$ and $10^{-12} \mathrm{~s}$, and neutron scattering is a powerful method to investigate biological matter. Indeed, it has been shown that MBP bound to oriented reconstituted myelin membranes increases the membrane flexibility significantly, in particular the out-of-plane lipid motions, ${ }^{39-41}$ whereas P2, upon binding to multi-lamellar vesicles (MLV), enhances membrane stability by reducing lipid dynamics. ${ }^{10}$

We recently started addressing the investigation, using neutron scattering techniques, of reconstituted membranes better mimicking PNS myelin, which contains both myelin proteins MBP and P2. ${ }^{42}$ These model membranes consist of two synthetic lipids (1,2-dioleoyl-sn-glycero3-phospho-L-serine [DOPS] and 1,2-dioleoyl-sn-glycero-3phosphocholine $[\mathrm{DOPC}]$ in a $1: 1 \mathrm{w} / \mathrm{w}$ ratio) with and without recombinant MBP and P2. DOPS is anionic with a molecular weight of $\mathrm{MW}=810 \mathrm{Da}$ and has a transition from the $\mathrm{L}_{\beta}$ gel phase to the $\mathrm{L}_{\alpha}$ liquid-crystalline phase at $\mathrm{T}_{\beta \rightarrow \alpha}$ $=262 \mathrm{~K}$, whereas DOPC is electrically neutral with $\mathrm{MW}=$ $786 \mathrm{Da}$ and $\mathrm{T}_{\beta \rightarrow \alpha}=253 \mathrm{~K}$. With 18 carbon atoms in each of the two hydrophobic lipid tails of DOPS and DOPC, the chain length is $22-29 \AA$ for both lipids. This synthetic lipid mixture is used to mimic the properties of the inner leaflet of the bilayers in myelin, which is negatively charged. Since the melting point (unfolding temperature of the protein) of human $\mathrm{P} 2$ is $335 \mathrm{~K},{ }^{14}$ DOPS and DOPC, due to their noticeably lower phase transitions, allow studying the effect of protein addition both in the gel and the liquid membrane states. ENS, QENS, and neutron diffraction experiments as a function of temperature were initiated to study structural and dynamical properties of such reconstituted membranes, across the gel to liquid phase transitions of both lipids.

In a first paper, ${ }^{42}$ we argued the membrane structural changes evidenced between the gel and the liquid lipid phases. The diffraction measurements revealed that in the liquid phase, the MBP- and P2-bound membranes show a slightly different structural behaviour from the protein-free membrane. These results suggest that the membranes with proteins may adopt two different structures in the liquid phase: one thicker domain with, and another thinner domain without, proteins (reflecting d-spacings of $\sim 59 \AA$ and $\sim 79 \AA$ for MBP; $\sim 65 \AA$ and $\sim 74 \AA$ for P2; $\sim 55 \AA$ and $\sim 65 \AA$ for the MBPP2 proteo-lipid complexes). However, in the gel phase, both protein-free and protein-bound lipids show a single structure with a d-spacing of $\sim 59 \AA$. This remark, together with indication of squeezed out MBP in membrane in the gel phase, referenced in the literature, ${ }^{43,44}$ suggests that MBP and P2 behave similarly and are squeezed out from the lipid domains in the gel phase. However, when adding both MBP and P2 proteins, their stacking effect ${ }^{7}$ results in more ordered lipid domains, with consequent recovered intensity in the diffraction pattern.

Moreover, in Ref. 42, we reported results on the membrane dynamical properties at room temperature, where the lipids are in the liquid lipid phase, using a combination of spectrometers worldwide available: IN5 (Institute LaueLangevin, ILL, France), Osiris (ISIS at the Rutherford Appleton Laboratory, Oxfordshire, United Kingdom), and Neat (Helmholtz-Zentrum Berlin, Berlin, Germany) having complementary energy resolutions. This allowed us to evidence no significative changes in membrane mobility upon protein addition. In particular, a model was proposed based on NMR available results: a slow restricted diffusion could be assigned to the initial part of the lipids, including the headgroups, until the double bond of the lipid chains, and a fast confined diffusion to the terminal part of the lipid chains. The radii of the confinement were found to be approximately $5 \AA$ for all samples and both types of diffusions, while a 2-site jump diffusion could be assigned to the hydrogen atoms in the methylene groups in the lipid chains, having a distance of approximately $1.8 \AA$ A.

In this paper, we extend the investigation to the low $\mathrm{T}$ regime through experiments performed on the backscattering spectrometers IN13 and IN16, on the time of flight 
spectrometer IN5 and on the small momentum transfer diffractometer D16 at ILL.

\section{MATERIALS AND METHODS}

\section{A. Sample preparation}

Recombinant His $_{6}$-tagged murine 18.5-kDa MBP and His $_{6}$-tagged human P2 were expressed and purified as described in Ref. 42.

DOPS and DOPC were purchased from Avanti $\AA$ Polar Lipids (Alabaster, USA) and used without further purification (purity of $>99 \%$ ). Unilamellar vesicles (ULV) were obtained as reported previously. ${ }^{42}$ Appropriate amounts of protein solution with a concentration, which demonstrated protein-lipid binding $\left(0.14 \mathrm{mg} / \mathrm{ml}\right.$ for $\mathrm{MBP}$ and $0.90 \mathrm{mg} / \mathrm{ml}$ for $\mathrm{P} 2$ in $\left.\mathrm{D}_{2} \mathrm{O}\right)$, were added to the ULV solution. The following samples were prepared: protein-free lipids, lipids with MBP, lipids with P2 and lipids with both MBP and P2. The protein content of the membrane sample was very little, which allowed to neglect the scattering signal arising from the proteins and to investigate the dynamics of the membrane only, in presence of the proteins.

Oriented bilayers were obtained by spreading the samples on mono-crystalline $\mathrm{SiO}_{2}\left(\begin{array}{lll}1 & 1 & 0\end{array}\right)$ wafers. The samples were dried in a vacuum oven with a gentle nitrogen stream at $40^{\circ} \mathrm{C}$ for $24 \mathrm{~h}$ and re-hydrated in a desiccator with a $\mathrm{D}_{2} \mathrm{O}$ atmosphere for $36 \mathrm{~h}$. The relative humidity was kept at $95 \%$ with a saturated $\mathrm{CaSO}_{4} \mathrm{D}_{2} \mathrm{O}$ solution. For neutron scattering experiments, 3 such wafers were stacked in a slab-shaped aluminium sample holder with an empty wafer on the top. To make it vacuum tight, an indium seal was used, when closing the sample holder.

\section{B. Sample characterization}

Lipid-protein binding was verified by sucrose gradient ultracentrifugation (using an SW41 rotor), followed by SDSPAGE gel electrophoresis. ${ }^{45}$ Dynamic light scattering (DLS) measurements were performed, using the $A L V C G S-3$ Compact Goniometer (ALV-GmbH, Germany), to estimate the average hydrodynamic radius $\left\langle R_{h}\right\rangle$ and the polydispersity index $P D I$ of the ULVs in solution with and without proteins (the lipid concentration was here $0.001 \mathrm{mg} / \mathrm{ml}) .\left\langle R_{h}\right\rangle$ and $P D I$ were calculated according to Ref. 46 (Table I). Since the $P D I$ is very small, the samples can be considered as monodisperse.

Differential scanning calorimetric (DSC) experiments were performed using a DSC-131 device (Setaram Instrumentation, France) to check the occurrence of the gel-to-liquid

TABLE I. Hydrodynamic radii $\left\langle R_{h}\right\rangle$ and polydispersity indices $P D I$ for the ULVs with and without proteins, obtained by DLS.

\begin{tabular}{lcccc}
\hline \hline & LIPIDS & MBP & P2 & MBP + P2 \\
\hline$\left\langle R_{h}\right\rangle^{\mathrm{a}}(\mathrm{nm})$ & $50(1)$ & $56(1)$ & $57(2)$ & $58(2)$ \\
$P D I^{\mathrm{b}}$ & $0.1162(3)$ & $0.1558(9)$ & $0.1096(7)$ & $0.145(1)$ \\
\hline
\end{tabular}

${ }^{\mathrm{a}}\left\langle R_{h}\right\rangle$ is given by the Stokes-Einstein equation.

${ }^{\mathrm{b}} P D I$ is defined by the ratio between the standard deviation $\sigma$ of the decay time $\tau$ for particles in Brownian motion and the average value $\langle\tau\rangle{ }^{46}$ lipid phase transitions at nominal temperatures known from the literature.

\section{Neutron scattering experiments}

To investigate the dynamical properties of the membranes, temperature dependent incoherent ENS scans were performed on the thermal and cold high resolution backscattering spectrometers IN $13^{47}$ and IN $16,{ }^{48}$ respectively, while QENS measurements at $230 \mathrm{~K}$ were acquired on the timeof-flight spectrometer IN5 ${ }^{49}$ at ILL.With an almost Qindependent energy resolution of $\Delta \mathrm{E}=8 \mu \mathrm{eV}$ full width at half maximum (FWHM) and an accessible momentum transfer range of $0.2 \leq \mathrm{Q} \leq 4.9 \AA^{-1}$, IN13 allows the investigation of molecular motions in the time-scale up to $100 \mathrm{ps}$ and with an amplitude from $1.3 \AA$ to $\sim 31 \AA$.

The cold backscattering spectrometer IN16 is situated on a guide looking at one of the cold sources of the ILL. The $\mathrm{Si}$ (111) reflection of the monochromator is used to select a wavelength of $\lambda=6.27 \AA$ among the incoming neutrons. The instrumental setup results in a very narrow elastic energy resolution of $0.9 \mu \mathrm{eV}$ (FWHM) corresponding to a time window of about $1 \mathrm{~ns}$. The momentum transfer range is here from 0.19 to $1.89 \AA^{-1}$.

IN5 is a disk chopper time-of-flight spectrometer which allows spanning a wide range of incident wavelengths and energy resolutions. Neutrons, scattered at the sample, are detected at $4 \mathrm{~m}$ distance by 259 spherically ordered ${ }^{3} \mathrm{He}$ detectors of the height of $3 \mathrm{~m}$, covering an angular range between $14.5^{\circ}$ and $132.5^{\circ}$. A position sensitive detector (PSD) counts neutrons in the small angle region between $2^{\circ}$ and $8^{\circ}$. The instrument was used at an incident wavelength of $10 \AA$, the energy resolution is $12 \mu \mathrm{eV}$ (FWHM) and the momentum transfer range $0.23 \AA^{-1}<\mathrm{Q}<1.14 \AA^{-1}$.

To investigate motions parallel and perpendicular to the membrane surface, samples were oriented at $135^{\circ}$ (in-plane direction) and $45^{\circ}$ (out-of-plane direction) with respect to the incoming neutron beam. On both instruments, IN16 and IN13, elastic scans were acquired over a large temperature range, $20-300 \mathrm{~K}$, in order to cover the lipid phase transitions. On IN13, each temperature point was measured for $2 \mathrm{~h}$ in order to obtain a high signal to noise ratio. On the other hand, on IN16 the sample temperature was varied following a linear ramp of $2 \mathrm{~K} / \mathrm{min}$ from 20 to $200 \mathrm{~K}$ and of $0.3 \mathrm{~K} / \mathrm{min}$ from 200 to $300 \mathrm{~K}$ with parallel acquisition of five minutes elastic runs. The sample thickness was properly chosen to minimize the neutron absorption from the sample (sample transmission $\sim 90 \%$ ), thus neglecting corrections for multiple scattering processes. For background corrections, the signal of an empty cell with wafers was subtracted from the raw data normalized to the neutron flux. The scattering signal of the lowest temperature run $(20 \mathrm{~K})$ provided the normalization factor for the detector efficiency. To describe the intra-molecular dynamics, the atomic mean square displacements, $(\mathrm{MSD}=$ $\left\langle u^{2}\right\rangle(T)$ ) have been calculated. Therefore, the elastic part of the scattering function,

$$
S(Q, \omega)=\frac{1}{2 \pi} \int_{-\infty}^{+\infty} I(Q, t) \cdot e^{-i \omega t} d t,
$$


can be described by a Gaussian approximation in the form of a Debye-Waller factor, ${ }^{50}$

$$
S(Q, \omega=0) \propto \exp \left(-\frac{1}{6}\left\langle u^{2}\right\rangle Q^{2}\right),
$$

which is approximately valid for $\left\langle u^{2}\right\rangle Q^{2} \lesssim 2$. $\mathrm{I}(\mathrm{Q}, \mathrm{t})$ is hereby the intermediate scattering function, which is the Fourier transform of $\mathrm{S}(\mathrm{Q}, \omega)$ in time. $\left\langle u^{2}\right\rangle$ can be obtained by linear regression in a semi-logarithmic plot of the scattering intensities as a function of $\mathrm{Q}^{2}$ for every measured temperature. For that, in agreement with a paper from Lehnert et al., ${ }^{51}$ a Q-range of $1.61 \AA^{-1} \mathrm{Q}^{2}<4.26 \AA^{-1}$ was chosen for the fitting, to lie within the Gaussian approximation limit (linear slope of the logarithmic intensities).

The QENS data at $230 \mathrm{~K}$ were analysed in terms of sets of two hydrogen populations performing three different motions:

(i) A population $f$ executing only vibrational motions described by a Debye-Waller factor $e^{-\left\langle u^{2}\right\rangle Q^{2}}$ and that appears immobile within the instrumental resolution;

(ii) A population $p_{V \otimes R o t}$ that diffuses in a confined spherical volume of radius $R_{V}$ with diffusion constant $D_{V}$ described by the Volino-model ${ }^{52} S_{V}(\vec{Q}, \omega)$, and that also rotates isotropically following the scattering law described by the Sears-model ${ }^{53} S_{R o t}(\vec{Q}, \omega)$.

The resulting total scattering function describing data obtained at $230 \mathrm{~K}$ is thus,

$$
\begin{aligned}
S_{\text {theo }}(\vec{Q}, \omega)= & e^{-\left\langle u^{2}\right\rangle Q^{2}}\left[f \delta(\omega)+p_{V \otimes R o t}\left(S_{V}(\vec{Q}, \omega)\right.\right. \\
& \left.\left.\otimes S_{R o t}(\vec{Q}, \omega)\right)\right]
\end{aligned}
$$

with $f+p_{V \otimes R o t}=1$.

Neutron diffraction experiments were performed on the small momentum transfer diffractometer D $16^{54}(\lambda=4.75 \AA$, $0.04 \AA^{-1} \leq \mathrm{Q} \leq 0.42 \AA^{-1}$ ) at the ILL, in order to study the mosaic spread and the temperature evolution of the repeat distances of the membranes. The temperature scans ranged from $240 \mathrm{~K}$ to $300 \mathrm{~K}$ and back, covering the gel to liquid phase transition of the lipids. The mosaic spread $\Delta \omega$, an indication for the intrinsic mismatch of multilayer's perfect parallelism, was obtained by rotation of the sample with respect to the incoming beam and was calculated from the FWHM of the first order Bragg peak as a function of $\omega$ (example shown in Figure 1). For that, a narrow and a broad Lorentzian have been used to fit the data points. The narrow Lorentzian describes the mosaic spread and the broad one probably fluctuations like disorder in the in-plane direction. $\Delta \omega$ was found to be less than $0.5^{\circ}$ for all samples. Considering several thousands $(\sim 6600)$ of bilayers per wafer, this low mosaicity confirms that the bilayers are highly parallel oriented, which is an important indication for the quality of the reconstituted membranes.

\section{RESULTS}

The Q-decay of the elastic intensity acquired on IN13 at different temperatures is shown in Figure 2.

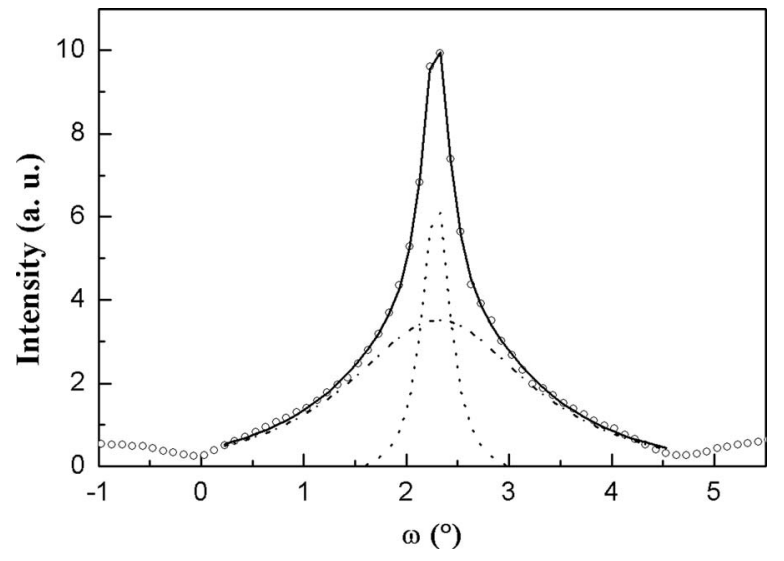

FIG. 1. Mosaic spread $\Delta \omega$ of the protein-free lipids at $300 \mathrm{~K}$ calculated from FWHM of the first order Bragg peak as a function of $\omega\left(\Delta \omega=0.328^{\circ}\right.$ $\left.\pm 0.009^{\circ}\right)$

Faster decay reflects higher dynamics promoted at higher temperatures, as expected. Two main features may be observed: while up to $250 \mathrm{~K}$ the decrease in intensity is nearly regular in T, above $250 \mathrm{~K}$ a faster decrease is observed and a first main gap in the intensity is achieved between $260 \mathrm{~K}$ and $270 \mathrm{~K}$ followed by a second gap between $270 \mathrm{~K}$ and $280 \mathrm{~K}$. Above this temperature, the proton dynamics of the sample is enhanced, the timescale window falling out of the instrumental resolution of IN13. Those two gaps are assigned to the lipid phase transition, from gel to liquid phase, and to the melting of the crystallized internal water molecules, respectively.

Figure 3 shows the temperature dependence of the normalized elastic intensities $\mathrm{I}(\mathrm{Q})$ measured on IN13 and integrated over the Q-range $0.5 \AA^{-1} \leq \mathrm{Q} \leq 4.5 \AA^{-1}$ for the inplane configuration of the protein-free lipids. The lower $\mathrm{Q}$ region $\left(0.2 \AA^{-1}<\mathrm{Q}<0.5 \AA^{-1}\right)$ was discarded accounting for Bragg peaks originating from membrane periodicity. DSC graphs showing the phase transitions of the lipids (top) and of the water between the bilayers (bottom) are reported for comparison in the insets of the figure.

The curve is characterized by four different regimes, enumerated in Figure 3 by $1-4$. In regime 1 , up to $\sim 150 \mathrm{~K}$, the system obeys to a harmonic regime of motions, resulting in a

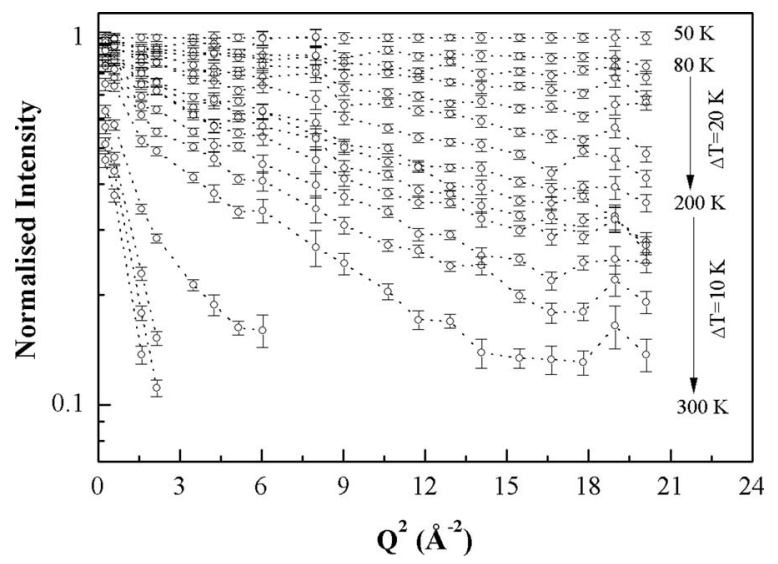

FIG. 2. Normalized elastic intensities of in-plane lipids $v s$. the momentum transfer Q, measured on IN13 as function of the temperature $(50-300 \mathrm{~K}$ from top to bottom). 


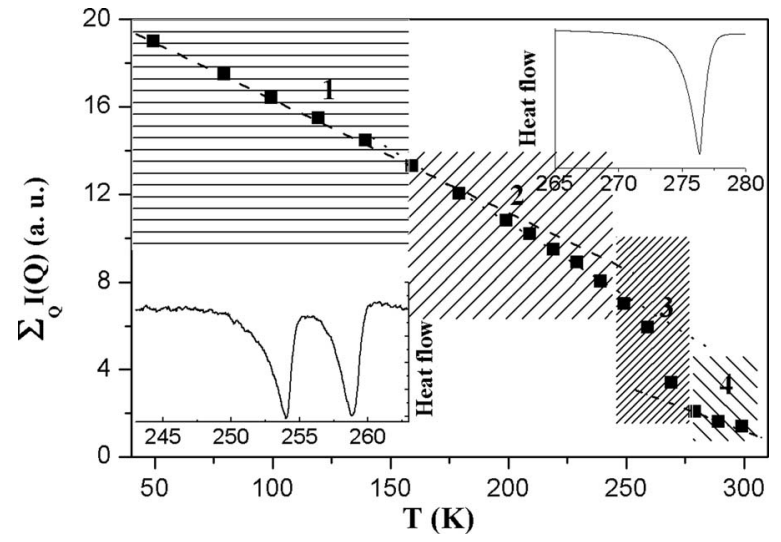

FIG. 3. Temperature dependent normalized elastic intensities of the lipids in the in-plane configuration measured on IN13 and integrated over a Q-range of $0.5 \AA^{-1} \leq \mathrm{Q} \leq 4.5 \AA^{-1}$. (Insets) DSC spectra showing the phase transitions of the lipids (bottom) and of the water between the bilayers (top).

linear trend of the temperature dependence of the scattering intensity. The appearance of new degrees of freedom confers to the membranes the ability to perform anharmonic motions (region 2). In particular, at $\mathrm{T} \sim 160-180 \mathrm{~K}$ the transition from harmonic to anharmonic regimes occurs regardless of the specific lipid composition. Similar behaviour has previously been found by other research groups by ENS measurements on purple membranes ${ }^{55-57}$ and proteins ${ }^{58,59}$ at $\mathrm{T} \sim 150 \mathrm{~K}$. This transition has been attributed to methyl group $\left(\mathrm{CH}_{3}\right.$-molecules $)$ rotations with characteristic rotational diffusion rates entering in the experimental time-window of the instrument.

In regime 4 , for $\mathrm{T} \geq 280 \mathrm{~K}$, the membrane is in the liquid phase and the internal water is free promoting high lipid dynamics. Here, the almost flat temperature dependence of the integrated elastic intensities, which is in agreement with the gap shown in Figure 2, confirms that complementary experiments are necessary. A broader energy resolution and a lower accessible Q range would allow investigating the membrane dynamics in this temperature region more precisely. More peculiar is the strong variation of the intensity observed at $250 \mathrm{~K}<\mathrm{T}<280 \mathrm{~K}$, indicating a fast change in the lipid dy-

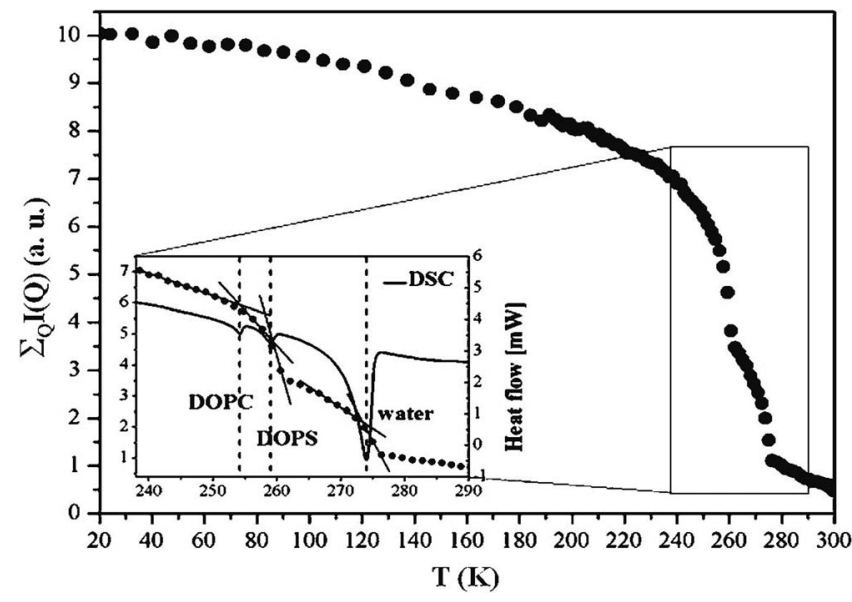

FIG. 4. Temperature dependence of the integrated elastic intensities of the protein-free lipids in the in-plane configuration measured on IN16. A magnification of the intensity for a temperature ranging from about $240 \mathrm{~K}$ to $290 \mathrm{~K}$ is shown in the inset and compared to a DSC graph. namics (region 3), which occurs at both orientations. Region 3 is the temperature region where the gel to liquid phase transition occurs for both DOPC and DOPS as shown by a DSC measurement (Figure 3, down inset). Moreover, the membrane being fully hydrated ( $h=95 \%)$, free water molecules are also present, thus changes associated to the water melting point, that occurs at $\sim 276 \mathrm{~K}$ for $\mathrm{D}_{2} \mathrm{O}$, as shown in the DSC graph (Figure 3, top inset), are expected. Thus, care should be taken when discussing region 3 . However, we remind that the use of heavy water to hydrate the membranes allows us to neglect the pure water contribution to the global dynamics, thanks to the very low incoherent scattering cross section of deuterium compared to hydrogen $\left(\sigma_{\text {inc }}=2\right.$ and 80 barn for $\mathrm{D}$ and $\mathrm{H}$, respectively). Hence, with this technique we also monitor changes in membrane dynamics induced by enhancement/reduction of water mobility. These systems seem thus to be characterized by three main transitions (DOPC, DOPS, and water melting) that, occurring in a restricted temperature region $250<\mathrm{T}<280 \mathrm{~K}$, cannot be properly resolved separately. To better investigate this interesting region, we report in Figure 4 the temperature dependence of the $\Sigma_{\mathrm{Q}} \mathrm{I}(\mathrm{Q})$ $\left(0.45 \AA^{-1}<\mathrm{Q}<1.49 \AA^{-1}\right)$ measured on IN16 (bottom panel), together with the DSC graph measured on the same systems. As for IN13, the low $Q$ values $\left(\mathrm{Q}<0.45 \AA^{-1}\right)$ have been discarded.

The previously found gaps in intensities are still observed in the same temperature regions while the two distinct effects of the lipid and water phase transitions on the lipid dynamics are now well resolved. It is indeed clear that IN16 allows to better distinguishing the two contributions. This might arise either from a better signal-to-noise ratio (which leads to the optimization between the acquisition time and the T-ramp) achievable on IN16 with respect to IN13, which is directly linked to the instrument neutron flux at the sample, or to the given energy resolution. The latter would imply that the water melting and the lipid phase transition affect motions spanning time-scales typically covered by IN16 and only partially by IN13.

The enhanced dynamics in the high $\mathrm{T}$ region are also maintained, as demonstrated by the less pronounced dependence of the elastic intensities upon the temperature (see Figure 4, bottom panel). Using Eq. (2), the MSD can be calculated. Results are reported in Figure 5 for the in-plane dynamics of the protein-free lipids, obtained from IN13 (upper panel) and IN16 (bottom panel) data. The solid lines are guides to the eyes.

A strong increase in the MSD is then associated with the occurrence of the lipid phase transitions. We remind that, due to the H-D isotope exchange in the membrane hydration water, the water melting point is shifted by a few degrees $\left(\Delta \mathrm{T}=3.82{ }^{\circ} \mathrm{C}\right.$, i.e., $\left.\mathrm{T}_{\mathrm{D}_{2} \mathrm{O}}=276.8 \mathrm{~K}\right)$.

The melting of free water molecules induces flexibility to the lipid membrane, by enhancing its proton dynamics. We underline that the MSD obtained for the high $\mathrm{T}$ region $(\mathrm{T}>280 \mathrm{~K})$ are presented only for the sake of completeness, being the limit for the validity of the Gaussian approximation not respected.

Thus, at these energy resolutions, changes in the lipid dynamics induced by membrane anisotropy and/or protein 


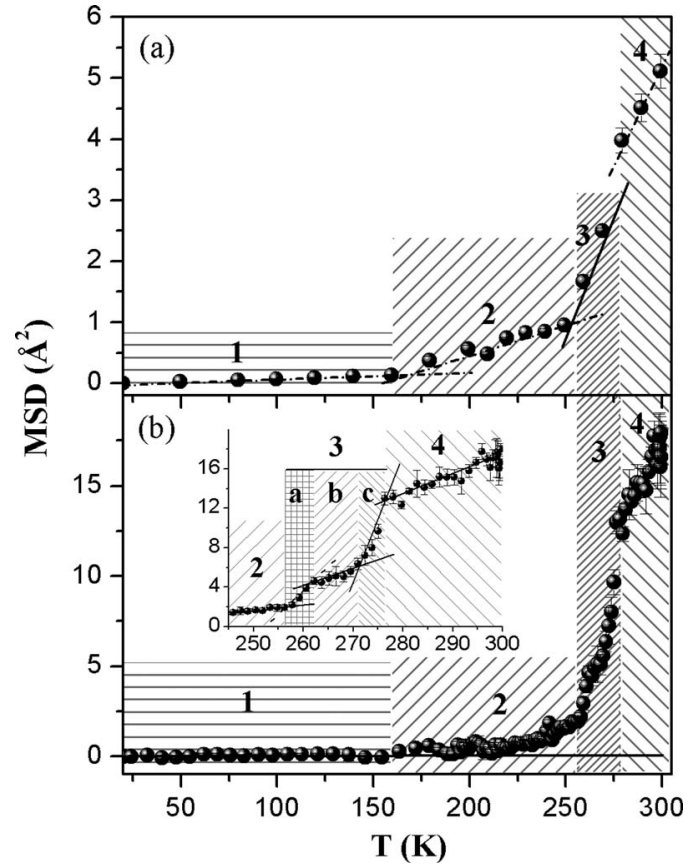

FIG. 5. MSD as a function of the temperature obtained from IN13 (a) and IN16 (b) data.

addition might be unambiguously observed only in the $\mathrm{T}$ region below $280 \mathrm{~K}$.

To investigate, whether such temperature-dependent features of proton dynamics reflect structural changes of the membrane, the temperature region across the lipid-phase transition has been carefully explored on D16. Figure 6 shows the elastic scattered intensity as a function of the momentum transfer Q for the protein-free lipids, measured at $246 \mathrm{~K}$, i.e., in the gel phase. The diffraction pattern clearly shows at least the first 3 orders of reflections, with a periodicity typically observed for hydrated model membranes (59 ̊).

The diffraction pattern of the protein-free lipids was then monitored continuously during the heating of the sample from 240 to 290 K. In Figure 7, we report the shift in Q of the position of the maximum of the first Bragg reflection induced by the temperature increase. A clear shift of the Bragg peak is observed at intermediate temperatures, while structures at low and at room temperature do not show changes in the peak

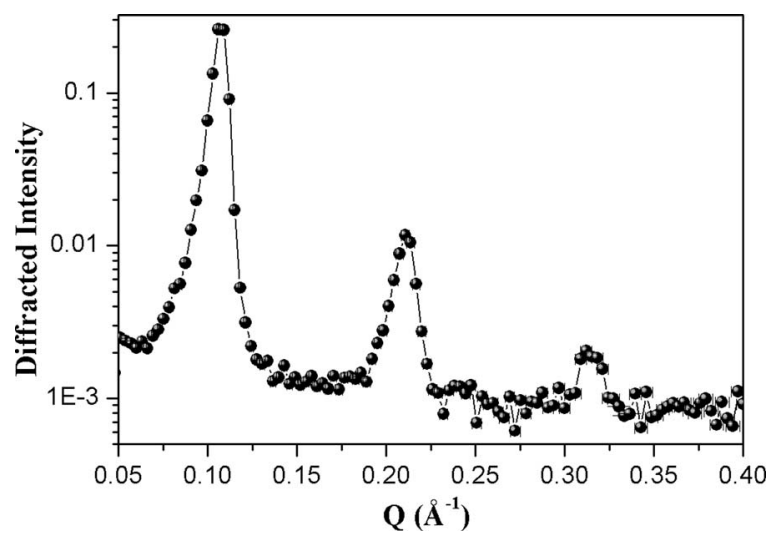

FIG. 6. Diffraction pattern of protein-free membrane measured at $246 \mathrm{~K}$ on D16.

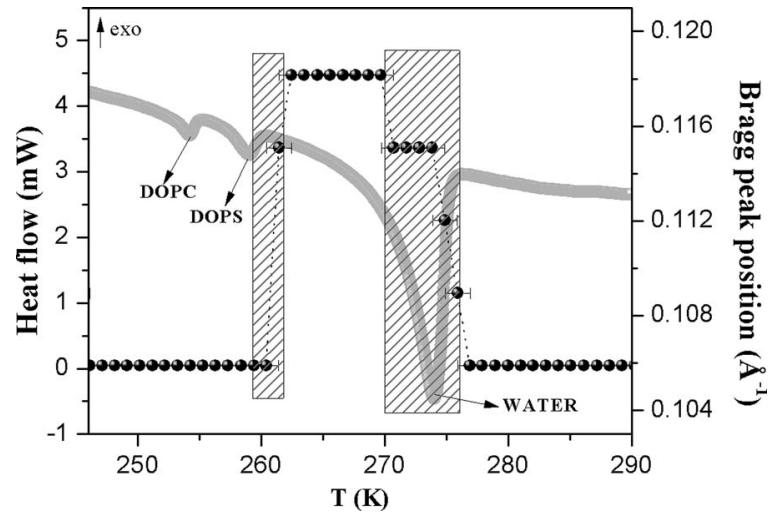

FIG. 7. Protein-free membrane DSC graph compared to the shift in temperature of the position of the first Bragg reflection.

position. The decrease of the Bragg peak position, meaning an increase in d-spacing, during the melting process of water corresponds to a known effect: when membranes are cooled down a decrease in lamellar spacing has been identified due to water leaving the inter-membrane space to form crystalline ice outside the regular stack of membranes. ${ }^{56,60}$ Upon subsequent heating, the lamellar spacing first remains constant and then increases between $260 \mathrm{~K}$ and room temperature to recover the initial value as the ice melts and water flows back into the membrane stack.

In particular, three key temperature regions are evidenced, representing the regimes below the lipid phase transition $(\mathrm{T}<261 \mathrm{~K})$, above lipids are both in the liquid phase but well below the water melting $(262 \mathrm{~K}<\mathrm{T}<276 \mathrm{~K})$, and above the melting of the water molecules $(\mathrm{T}>276 \mathrm{~K})$. Thus, the figure suggests that a particular behaviour may occur in the temperature region at which changes in membrane dynamics were also observed. For that, the comparison with a DSC graph of the same sample shows a strict correspondence between the first order Bragg peak shift and the phase transitions. More in detail, the lipid phase transition of the neutral lipid DOPC ( $\mathrm{T}_{\beta \rightarrow \alpha}=253 \mathrm{~K}$ ) is not sufficient to impact the membrane structure, while strong structural variations occur after also the anionic DOPS lipid $\left(\mathrm{T}_{\beta \rightarrow \alpha}=262 \mathrm{~K}\right)$ phase transition has taken place.

We now address the question, whether the addition of myelin proteins to lipids may affect the dynamical properties of proton mobility in the sample, while keeping its structure unchanged in the gel phase as reported in Ref. 42. For that, we will mainly focus on the temperature region below the water melting point. In Figure 8 we report the temperature dependence of the in-plane (a) and out-of-plane (b) MSD of proteinfree lipids (full circles), lipids $+\mathrm{MBP}$ (triangles), lipids $+\mathrm{P} 2$ (squares), and lipids $+\mathrm{MBP}+\mathrm{P} 2$ (rhomboids) obtained from IN13 data. The solid line represents the harmonic contribution to the MSD described in terms of a set of quantized Einstein harmonic oscillators. ${ }^{61,62}$ Data are shown for $\mathrm{T}<300 \mathrm{~K}$ not obeying the limit for the applicability of the Gaussian approximation at the highest temperature points. In the inplane configuration, all samples exhibit a deviation from the harmonic regime at $\mathrm{T} \geq 180 \mathrm{~K}$. However, the lipid $+\mathrm{MBP}$ sample conserves reminiscences of harmonic motions for a 


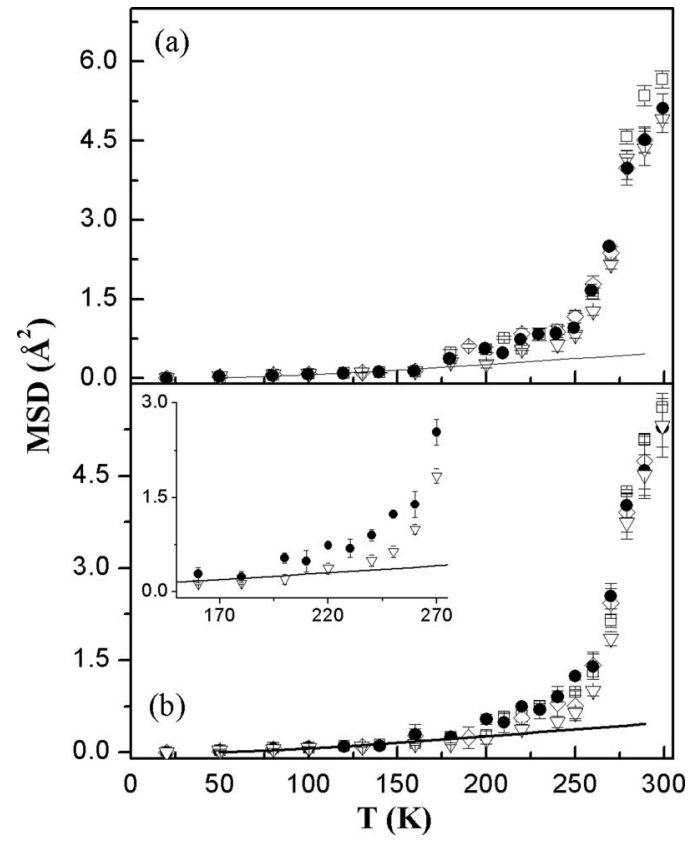

FIG. 8. Temperature dependence of the in-plane (a) and out-of-plane (b) MSDs of protein-free lipids (full circles), lipids + MBP (triangles), lipids $+\mathrm{P} 2$ (squares), and lipids $+\mathrm{MBP}+\mathrm{P} 2$ (rhomboids). The solid line represents the harmonic behaviour. (Inset) Magnification of the behaviour of free-lipids and lipids + MBP in the 150-270 K temperature region.

few degrees more. In contrast, for the out-of-plane configuration the situation changes. Although only minor modifications in the membrane dynamics are observed when P2 is present as well as in case of the simultaneous addition of both proteins, major modifications are induced by the addition of MBP. The simultaneous addition of both proteins results in an intermediate situation. This is clearly visible in the inset of Figure 8(b), where the interesting temperature region is magnified for protein-free lipids and lipids + MBP. The lipids + MBP behave harmonically up to $\mathrm{T} \geq 240 \mathrm{~K}$, while the protein-free lipids are more flexible at $\mathrm{T} \geq 180 \mathrm{~K}$, as in the in-plane geometry.

The reduction in membrane mobility lasts until both the gel-to-liquid lipid phase transitions are completed. Curiously, this finding is the opposite to previous results ${ }^{39,40}$ for charged model membranes, where we demonstrated that the addition of 5\% (w/w) MBP to acidic lipid dimyristoyl L- $\alpha$ phosphatidic acid (DMPA) bilayers highlighted the enhancement of out-of-plane dynamics of the membrane above the lipid phase transition. This effect could be due to the increased mobility of the lipid alkyl chains, caused by melting of interlayer water molecules. ${ }^{39}$ Thus, the presence of the neutral DOPC lipids, accounting half of the total lipid fraction, probably plays a key role here. Also the lipid shape may have an effect on the role of the protein on the membrane dynamics. Indeed, in contrast to what was observed here, P2 was previously found to enhance the stability of myelin-like vesicles made of DMPA and DOPS-DOPC lipids, respectively, reducing their dynamics. ${ }^{10}$

The membrane dynamical behaviour in the gel lipid phase was then investigated through QENS experiments performed at $230 \mathrm{~K}$ on the high energy resolution spectrometer

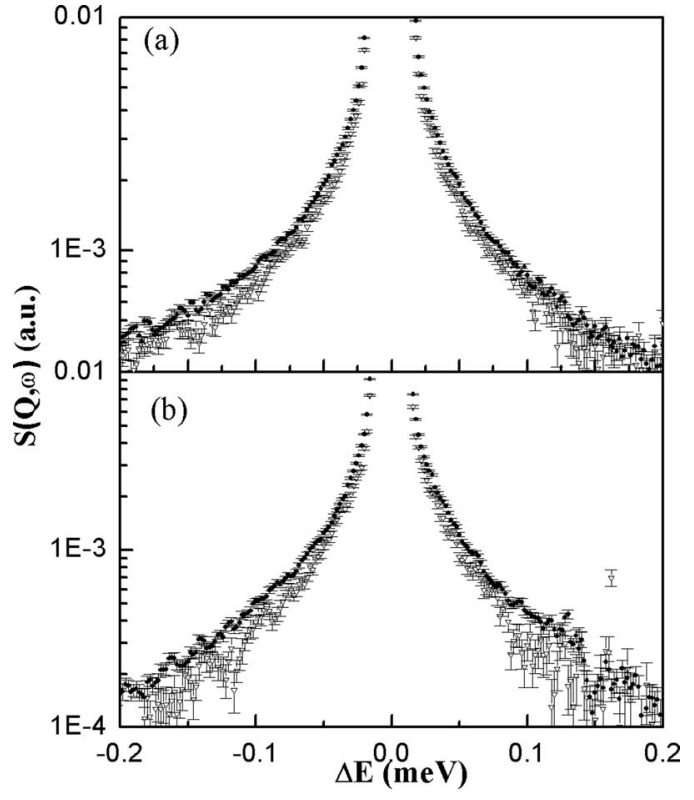

FIG. 9. In-plane (a) and out-of-plane (b) QENS spectra of protein-free lipids (full circles) and lipids + MBP (triangles) measured at $230 \mathrm{~K}$ on IN5. Data are binned over the whole accessible $\mathrm{Q}$ range. The curves are normalized to unity at the maximum of the elastic peak and only the lower parts are shown to highlight the quasi-elastic broadening.

IN5, using approximately the same energy resolution as on IN13. In Figure 9 we report the in- and out-of-plane QENS spectra of protein-free lipids and lipids + MBP. Data corresponding to lipids $+\mathrm{P} 2$ and lipids $+\mathrm{MBP}+\mathrm{P} 2$ are not shown, as they are hardly distinguishable from the proteinfree lipid data. Data are binned over the whole accessible Q range.

In the gel lipid phase the elastic contribution of the scattering function is very high and the quasielastic contribution accounts for only few percents. Nevertheless, fitting the low T data of the protein-free membranes with (3), within the $10 \%$ of the hydrogen fraction that performs diffusion in confinement and simultaneously rotates isotropically, the radius of confinement is found in the out-of-plane configuration to be $6 \AA$, much larger than in the in-plane configuration ( $3 \AA$ ). The radii of rotation are estimated to be approximately $1.1 \AA$ for both configurations and can be attributed to the $\mathrm{C}-\mathrm{H}$ bond length of the methylene groups in the lipid chains. This suggests that in the gel phase of the lipids, the energy for the methylene groups is probably not high enough to perform jumps across two sites, but sufficient to rotate isotropically.

When comparing the samples, differences are also observed. The resulting fitting parameters for data obtained in the ordered gel phase of the lipids, at $230 \mathrm{~K}$, are shown in Table II for all the investigated samples.

The confined diffusion is slowed down in the outof-plane configuration of MBP-bound lipids, with respect to the other samples. This confirms the elastic data obtained on IN13, which indicated that the membrane behaves harmonically up to higher temperatures when MBP is added, and thus influences methyl group rotation. In contrast, the correlation time is found to be smaller for the MBP-containing lipids in the out-of-plane configuration, indicating that 
TABLE II. Fit parameters for data obtained at $230 \mathrm{~K}$.

\begin{tabular}{lcccccc}
\hline \hline SAMPLE & $\mathrm{R}_{\mathrm{V}}(\AA)$ & $\mathrm{D}_{\mathrm{V}}\left(\mathrm{cm}^{2} / \mathrm{s}^{-5}\right)$ & $\mathrm{R}(\AA)$ & $\tau\left(\mathrm{meV}^{-1}\right)$ & $f$ & $\mathrm{p}_{\mathrm{V} \otimes \mathrm{Rot}}$ \\
\hline Out-of-plane configuration & & & & & & \\
Protein-free lipids & 6 & 0.10 & 1.1 & 6.65 & 0.91 & 0.10 \\
Lipids + MBP & 9 & 0.06 & 1.1 & 1.07 & 0.89 & 0.11 \\
Lipids + P2 & 3 & 0.33 & 1.6 & 6.60 & 0.96 & 0.04 \\
Lipids + MBP + P2 & 3 & 0.77 & 2.2 & 4.00 & 0.96 & 0.04 \\
In-plane configuration & & & & & & \\
Protein-free lipids & 3 & 0.68 & 1.2 & 2.03 & 0.92 & 0.08 \\
Lipids + MBP & 9 & 0.02 & 2.0 & 9.35 & 0.92 & 0.09 \\
Lipids + P2 & 6 & 0.02 & 1.0 & 10.00 & 0.79 & 0.21 \\
Lipids + MBP + P2 & 8 & 0.02 & 1.0 & 9.86 & 0.79 & 0.21 \\
\hline \hline & & & & & & \\
The absolute errors are: $\Delta R_{v} \approx \pm 1 \AA, \Delta D v \approx \pm 0.010^{-5} \mathrm{~cm}^{2} / \mathrm{s}, \Delta R \approx \pm 0.5 \AA, \Delta \tau \approx \pm 0.01 \mathrm{meV}^{-1}$, and $\Delta f \approx \Delta p_{V \otimes r o t} \approx 0.05$.
\end{tabular}

rotation of the methylene groups with radii of rotation of 1.1 $\AA$ is faster, as already found for the lipids. For the in-plane configuration, no significant differences are observed for the two molecular motions.

\section{DISCUSSION}

Our results raise the question, how MBP and P2 bound to a lipid membrane affect its dynamics.

We have shown that:

i. Both lipids have to undergo the gel-to-liquid phase transitions to observe changes in the membrane structure;

ii. The addition of MBP and/or P2 affects the membrane structure in the liquid phase, while keeping the gel phase unchanged;

iii. Water melting affects the membrane structure significantly;

iv. The addition of MBP confers rigidity to the membrane, in particular stabilizing the out-of-plane lipid motion, allowing the protons to behave harmonically up to higher temperatures;

v. While elastic data acquired at room temperature could not be unambiguously exploited, QENS results reveal that MBP and P2 keep unchanged the membrane mobility in the liquid lipid phase;

vi. QENS results achieved at $230 \mathrm{~K}$ (gel lipid phase) confirm that the addition of MBP slightly reduces membrane mobility, confirming IN13 results. However, the acquired enhancement of membrane stability does not affect the membrane structure significantly, which does not show differences at this temperature with respect to the protein-free lipids, suggesting that the protein is squeezed out.

To explain these results, the properties of MBP and P2 bound to a membrane have to be understood. Sedzik et al. ${ }^{63,64}$ have estimated the lipid-bound conformations of MBP and $\mathrm{P} 2$, located between lipid bilayers, with X-ray diffraction. The width of inter-bilayer spaces in a multilayer with bound P2 was $33 \AA$ and with MBP only $23 \AA$, although MBP is larger. Mixed complexes with both proteins MBP and P2 exhibit an average space width between $23 \AA$ and $33 \AA$. Those interbilayer spaces are in agreement with a compact, globular structure for P2, located largely in the interbilayer spaces, with a small part possibly inserted into the lipid headgroup layers, ${ }^{65}$ whereas MBP seems to be flattened between the bilayers. It was also demonstrated that MBP causes single bilayer vesicles to create multilayers with MBP located between the bilayers and the interbilayer spacings are matchable to the cytoplasmic spaces in CNS myelin. ${ }^{66}$ Also P2 induced transformation of single bilayer vesicles into stable stacked multilayers, but only when the amount of P2 was larger than 4 wt. $\%$. The binding of P2 to lipids saturated at $15 \mathrm{wt} . \%$, corresponding to the amount of P2 in the PNS. Due to its high positive charge, MBP is extended in solution and is thus highly hydrophilic, resulting in an extensive interaction with negatively charged bilayers.

Suresh et al..$^{7}$ have further studied the effects of MBP and P2 on membrane bilayers with atomic force microscopy studies (AFM). Either MBP or P2 caused stacking of lipid bilayers, suggesting that they provide adhesive forces between the lipid stack. P2 induced membrane stacking at lower concentrations than MBP, possibly due to the local high positive charge of the $\mathrm{P} 2$ surface, as $\mathrm{P} 2$ is a compact, globular protein. In the presence of both proteins, this stacking effect was increased significantly, indicating that MBP and P2 are acting synergistically.

However, surprisingly, when MBP and P2 are bound together to the membrane (in the liquid lipid phase), it did not show strong differences in proton dynamics, while changing drastically the membrane structure.

\section{CONCLUSIONS}

Structural and dynamical properties of the proteo-lipid complexes show different behaviours upon addition of myelin proteins. In particular, in the lipid gel phase, the proton dynamics are reduced by the addition of MBP, while the membrane structure remains unchanged. On the other hand, in the liquid phase, strong structural variations occur in the membrane upon proteins addition, accompanied by only minor differences in membrane mobility.

\section{ACKNOWLEDGMENTS}

We would like to thank the ILL for fellowship and beam time, and the CSSB-HZI, DESY in Hamburg (Germany) for 
the use of the laboratories for protein purification. We thank B. Demé, J. Ollivier, and J. Combet for their distinguished help as local contacts during the neutron experiments. Furthermore, we are thankful to Iulia Blesniac and Juliette Devos, who helped during the sample characterisation and Ralf Schweins and I. Grillo for the DLS and DSC measurements, respectively.

${ }^{1}$ A. Peters, J. Anat. 98(1), 125 (1964).

${ }^{2}$ H. Webster, J. Cell Biol. 48, 348 (1971).

${ }^{3}$ N. Baumann and D. Pham-Dinh, Physiol. Rev. 81(2), 871 (2001).

${ }^{4}$ G. W. Brady, N. S. Murthy, D. B. Fein, D. D. Wood, and M. A. Moscarello, Biophys. J. 34, 345 (1981).

${ }^{5}$ R. H. Quarles, W. B. Macklin, and P. Morell, "Molecular, cellular and medical aspects," in Basic Neurochemistry, edited by S. Brady, G. Siegel, R. W. Albers, and D. Price (Academic Press Elsevier, New York, 2006), Chap. 4, pp. 51-71.

${ }^{6}$ S. Greenfield, S. Brostoff, E. H. Eylar, and P. Morell, J. Neurochem. 20(4), 1207 (1973).

${ }^{7}$ S. Suresh, C. Wang, R. Nanekar, P. Kursula, and J. M. Edwardson, Biochemistry 49, 3456 (2010).

${ }^{8}$ P. Riccio, L. Masotti, P. Cavatorta, A. De Santis, D. Juretic, A. Bobba, J. Pasquali-Ronchetti, and E. Quagliariello, Biochem. Biophys. Res. Commun. 134(1), 313 (1986).

${ }^{9}$ C. Readhead, B. Popkoa, N. Takahashia, H. D. Shinea, R. A. Saavedraa, R. L. Sidmana, and L. Hooda, Cell 48, 703 (1987).

${ }^{10}$ I. R. Bates, P. Matharu, N. Ishiyama, D. Rochon, D. D. Wood, E. Polverini, M. A. Moscarello, N. J. Viner, and G. Harauz, Protein Expression Purif. 20, 285 (2000).

${ }^{11}$ J. A. Benjamins and P. Morell, Neurochem. Res. 3, 137 (1978).

${ }^{12}$ B. Garbay, A. M. Heape, F. Sargueil, and C. Cassagne, Prog. Neurobiol. 61, 267 (2000)

${ }^{13}$ G. Harauz, N. Ishiyamaa, C. M. D. Hilla, I. R. Batesa, D. S. Libicha, and C. Farès, Micron 35, 503 (2004).

${ }^{14}$ V. Majava, C. Wang, M. Myllykoski, S. M. Kangas, S. U. Kang, N. Hayashi, P. Baumgärtel, A. M. Heape, G. Lubec, and P. Kursula, Amino Acids 39, 59 (2010).

${ }^{15}$ C. Wang, U. Neugebauer, J. Brück, M. Myllykoski, P. Baumgärtel, J. Popp, and P. Kursula, PLoS ONE 6(5), e19915 (2011).

${ }^{16}$ J. M. Boggs, G. Rangaraj, W. Gao, and Y.-M. Heng, Biochemistry 45, 391 (2006).

${ }^{17}$ G. Harauz, V. Ladizhansky, and J. M. Boggs, Biochemistry 48, 8094 (2009).

${ }^{18}$ J. K. Kim, F. G. Mastronardi, D. D. Wood, D. M. Lubman, R. Zand, and M. A. Moscarello, Mol. Cell. Proteomics 2, 453 (2003).

${ }^{19}$ Z. Spyranti, T. Tselios, G. Deraos, J. Matsoukas, and G. A. Spyroulias, Amino Acids 38, 929 (2010).

${ }^{20}$ R. E. Martenson, Myelin: Biology and Chemistry (CRC Press, Boca Raton, FL, 1992).

${ }^{21}$ P. Morell, R. H. Quarles, and W. T. Norton, "Myelin formation, structure and biochemistry," in Basic Neurochemistry (Raven, New York, 1994), pp. 117-143.

${ }^{22}$ B. D. Trapp, M. Dubois-Dalcq, and R. H. Quarles, J. Neurochem. 43(4), 944 (1984).

${ }^{23}$ A. Chmurzynska, J. Appl. Genet. 47(1), 39 (2006).

${ }^{24}$ V. Majava, E. Polverini, A. Mazzini, R. Nanekar, W. Knoll, J. Peters, F. Natali, P. Baumgärtel, I. Kursula, and P. Kursula, PLoS One 5, e10300 (2010).

${ }^{25}$ T. A. Jones, T. Bergfors, J. Sedzik, and T. Unge, EMBO J. 7(6), 1597 (1988).

${ }^{26}$ D. J. B. Hunter, R. Macmaster, A. W. Roszak, A. Riboldi-Tunnicliffe, I. R. Griffiths, and A. A. Freer, Acta Cryst. D61, 1067 (2005).

${ }^{27}$ R. A. Hughes, R. D. Hadden, N. A. Gregson, and K. J. Smith, J. Neuroimmunol. 100, 74 (1999).

${ }^{28}$ R. A. Hughes and D. R. Cornblath, Lancet 366, 1653 (2005).

${ }^{29}$ E. Polverini, A. Fasano, F. Zito, P. Riccio, and P. Cavatorta, Eur. Biophys. J. 28, 351 (1999).

${ }^{30}$ H. Haas, C. L. Oliveira, I. L. Torriani, E. Polverini, A. Fasano, G. Carlone, P. Cavatorta, and P. Riccio, Biophys. J. 86, 455 (2004).
${ }^{31}$ D. R. Beniac, M. D. Luckevich, G. J. Czarnota, T. A. Tompkins, R. A. Ridsdale, F. P. Ottensmeyer, M. A. Moscarello, and G. Harauz, J. Biol. Chem. 272, 4261 (1997).

${ }^{32}$ R. A. Ridsdale, D. R. Beniac, T. A. Tompkins, M. A. Moscarello, and G. Harauz, J. Biol. Chem. 272, 4269 (1997).

${ }^{33}$ P. Riccio, F. Zito, A. Fasano, G. M. Liuzzi, F. Lolli, E. Polverini, and P. Cavatorta, NeuroReport 9, 2769 (1998).

${ }^{34}$ L. S. Chia, J. E. Thompson, M. A. Moscarello, Proc. Natl. Acad. Sci. U.S.A. 81, 1871 (1984).

${ }^{35}$ Y. Hu, D. Wood, M. Moscarello, C. Husted, C. Genain, J. A. Zasadzinski, and J. Israelachvili, Proc. Natl. Acad. Sci. U.S.A. 101(37), 13466 (2004).

${ }^{36}$ W. Pfeiffer, T. Henkel, E. Sackmann, W. Knoll, and D. Richter, Europhys. Lett. 8, 201 (1989).

${ }^{37}$ W. Doster, S. Cusack, and W. Petry, Nature (London) 337, 754 (1989).

${ }^{38}$ F. Natali, A. Gliozzi, R. Rolandi, A. Relini, P. Cavatorta, A. Deriu, A. Fasano, and P. Riccio, Appl. Phys. A 74, 1583 (2002).

${ }^{39}$ F. Natali, A. Relini, A. Gliozzi, R. Rolandi, P. Cavatorta, A. Deriu, A. Fasano, and P. Riccio, Chem. Phys. 292, 455 (2003).

${ }^{40}$ F. Natali, A. Relini, A. Gliozzi, R. Rolandi, P. Cavatorta, A. Deriu, A. Fasano, and P. Riccio, Physica B 350, e623 (2004).

${ }^{41}$ W. Knoll, F. Natali, J. Peters, R. Nanekar, C. Wang, and P. Kursula, Spectroscopy 24(6), 585 (2010).

${ }^{42}$ W. Knoll, J. Peters, P. Kursula, Y. Gerelli, J. Ollivier, B. Demé, M. Telling, E. Kemner, and F. Natali, Soft Matter 10, 519 (2014).

${ }^{43}$ R. G. Oliveira and B. Maggio, Biochim. Biophys. Acta 1561, 238 (2002).

${ }^{44}$ C. M. Rosetti and B. Maggio, Biophys. J. 93, 4254 (2007).

${ }^{45} \mathrm{~J}$. Knol, "Membrane reconstitution and functional analysis of a sugar transport system," Ph.D. dissertation (University of Groningen, Germany, 1999).

${ }^{46}$ I. N. Bronstein, K. A. Semendjajew, G. Musiol, and H. Muhlig, Taschenbuch der Mathematik (Verlag Harri Deutsch, 2001).

${ }^{47}$ F. Natali, J. Peters, D. Russo, S. Barbieri, C. Chiapponi, A. Cupane, A. Deriu, M. T. Di Bari, E. Farhi, Y. Gerelli, P. Mariani, A. Paciaroni, C. Rivasseau, G. Schiro, and F. Sonvico, Neutron News 19(4), 14-18 (2008)

${ }^{48}$ B. Frick, A. Magerl, Y. Blanc, and R. Rebesco, Physica B 234-236, 11771179 (1997).

${ }^{49}$ J. Ollivier, M. Plazanet, H. Schober, and J. C. Cook, Physica B 350(1-3), 173-177 (2004).

${ }^{50}$ M. Ferrand, A. J. Dianoux, W. Petry, and G. Zaccai, Proc. Natl. Acad. Sci. U.S.A. 90, 9668 (1993).

${ }^{51}$ U. Lehnert, V. Réat, M. Weik, G. Zaccai, and C. Pfister, Biophys. J. 75, 1945 (1998).

${ }^{52}$ F. Volino and A. J. Dianoux, Mol. Phys. 41(2), 271 (1980).

${ }^{53}$ V. F. Sears, Can. J. Phys. 44, 1299 (1966).

${ }^{54} \mathrm{See}$ http://www.ill.eu/instruments-support/instruments-groups/instruments/ d16 for the description of the technical characteristics and scientific applications of the instrument D16 at ILL.

${ }^{55}$ K. Wood, M. Plazanet, F. Gabel, B. Kessler, D. Oesterhelt, D. J. Tobias, G. Zaccai, and M. Weik, Proc. Natl. Acad. Sci. U.S.A. 104(46), 18049 (2007).

${ }^{56}$ M. Weik, U. Lehnert, and G. Zaccai, Biophys. J. 89(5), 3639 (2005).

${ }^{57}$ K. Wood, D. J. Tobias, B. Kessler, F. Gabel, D. Oesterhelt, F. A. A. Mulder, G. Zaccai, and M. Weik, J. Am. Chem. Soc. 132(14), 4990 (2010).

${ }^{58}$ G. Schiro, C. Caronna, F. Natali, and A. Cupane, J. Am. Chem. Soc. 132(4), $1371(2010)$

${ }^{59}$ J. H. Roh, V. N. Novikov, R. B. Gregory, J. E. Curtis, Z. Chowdhuri, and A. P. Sokolov, Phys. Rev. Lett. 95(3), 38101 (2005).

${ }^{60}$ R. E. Lechner, J. Fitter, N. A. Dencher, and T. Hauss, J. Mol. Biol. 277, 593 (1998)

${ }^{61}$ G. E. Deibler, R. E. Martenson, and M. W. Kies, Prep. Biochem. 2(2), 139 (1972).

${ }^{62}$ M. Bée, Quasielastic Neutron Scattering (Adam Hilger, Bristol, 1998).

${ }^{63}$ J. Sedzik, A. E. Blaurock, and M. Hoechli, J. Mol. Biol. 174, 385 (1984).

${ }^{64}$ J. Sedzik, A. E. Blaurock, and M. Hoechli, J. Neurochem. 45(3), 844 (1985).

${ }^{65}$ H. Han, M. M. Myllykoski, S. Ruskamo, C. Wang, and P. Kursula, BioFactors 39(3), 233 (2013).

${ }^{66}$ I. R. Bates, J. B. Feix, J. M. Boggs, and G. Harauz, J. Biol. Chem. 279, 5757 (2004). 